\title{
Analysis on the Development of Intellectual Property Rights in Tianjin
}

\author{
Ying Cheng ${ }^{1}$, Juanxia Zhao ${ }^{1, *}$ Chunhong Zhu ${ }^{1}$ and Minghao Wang ${ }^{2}$ \\ ${ }^{I}$ School of Economics and Management, Tiangong University, Tianjin, China \\ ${ }^{2}$ Innovation and Entrepreneurship Development Center, Tianjin Sino-German University of Applied Sciences, Tianjin, \\ China \\ *Corresponding author. Email: bluecar2000@163.com
}

\begin{abstract}
\section{INTRODUCTION}

The "Tianjin Three-Year Action Plan for Science and Technology Innovation (2020-2022)" points out the development path for Tianjin how to enhance its ability of independent innovation and original innovation in the next three years, which provides not only strong support for building an important source of independent innovation but also the main source of original innovation in Beijing, Tianjin and Hebei. This paper analyzed the accumulation of Patents according to the effective ranking of patents, and gave the specific situation of patent sustainable development.
\end{abstract}

This paper used comparative analysis to study the development of patent application data, patent authorization rate and patent validity in Tianjin. Since 2011, there has been a large gap in the number of patent applications for invention, utility model and design in Tianjin. From the subject of application, industrial enterprises are the subject of patent application in Tianjin. The protection of intellectual property in Tianjin has achieved some results, but there are still some deficiencies. The level of patent transformation and application in Colleges and universities is relatively low. There is a lack of a unified intellectual property system between Beijing, Tianjin and Hebei regions. As a result, intellectual property rights face low infringement cost and high rights protection costs. This conclusion shows that it is necessary to build a unified intellectual property system, strengthen the protection of intellectual property policy and the implementation of patent supporting policies, and safeguard intellectual property owners' legitimate rights and interests.

Keywords: patent applications; patent authorization rate; patent effective amount; innovation

\section{CURRENT SITUATION OF INTELLECTUAL PROPERTY CREATION IN TIANJIN}

\subsection{Analysis of patent application amount}

Before 2008, the number of various patent applications in Tianjin developed steadily at a low level, and the number of patent applications for invention, utility model and design were comparable. Since 2011, there has been a big gap in the number of three types of patent applications, including the rapid growth of utility model patents and

\begin{abstract}
invention patents. On the contrary, after a slight decline, the number of applications was the same as that in the original year, and remained at that level for a long time. By 2019 , the number of patent applications in China had declined for the first time in nearly 24 years, with 24574 invention patent applications, a decrease of 2087 compared with 2018 , a year-on-year decrease of $7.83 \%$. To Tianjin, the number of invention patent applications also decreased by $7.83 \%$ yearly, which is due to China's efforts to optimize the application structure and improve the application quality in terms of the overall supervision of patent applications in 2018. Tianjin has formulated the implementation plan of strict intellectual property protection in Tianjin (2018-2020), implemented the nine actions of the strictest intellectual property protection, severely cracked down on intellectual property infringement, purified the overall environment of intellectual property and improved the purity of patents to a certain extent.
\end{abstract}




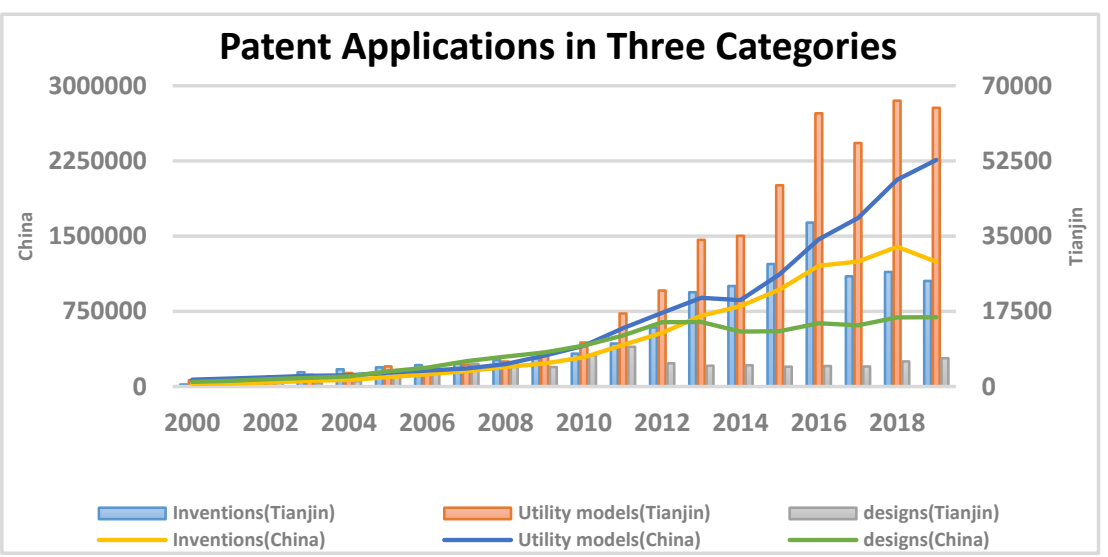

Figure 1. Patent Applications of Three Types of Patents in Tianjin and China

\subsection{Analysis of patent application subjects}

Among all types of patent applicants in Tianjin, industrial and mining enterprises account for the largest proportion. As a typical industrial city, industrial enterprises made great contributions to the local economy, and were the main body of patent applications. Colleges and universities have surpassed individuals and become the second subject of patent application since 2015. However, compared with enterprises, there is still a large gap. The number of patent applications of scientific research institutes and institutions has been at the bottom for a long time, which is far less than that of industrial and mining enterprises. As shown in
Figure 2, the annual patent application of various applicants entered a growth period after 2008, among which the enterprise increased most obviously. And the gap with other applicants began to widen. After experiencing a high-speed growth stage, patent applications of enterprises showed an inflection point in 2017, and the number of patent applications decreased significantly. This is due to the strict protection of intellectual property rights in Tianjin and the integration of intellectual property rights in Beijing, Tianjin and Hebei. The introduction of the policy has dealt a serious blow to patent infringement. Patent applications began to pick up again gradually in 2018, but at a much lower rate of growth, and the level of patent applications has been steady in the two years since then.

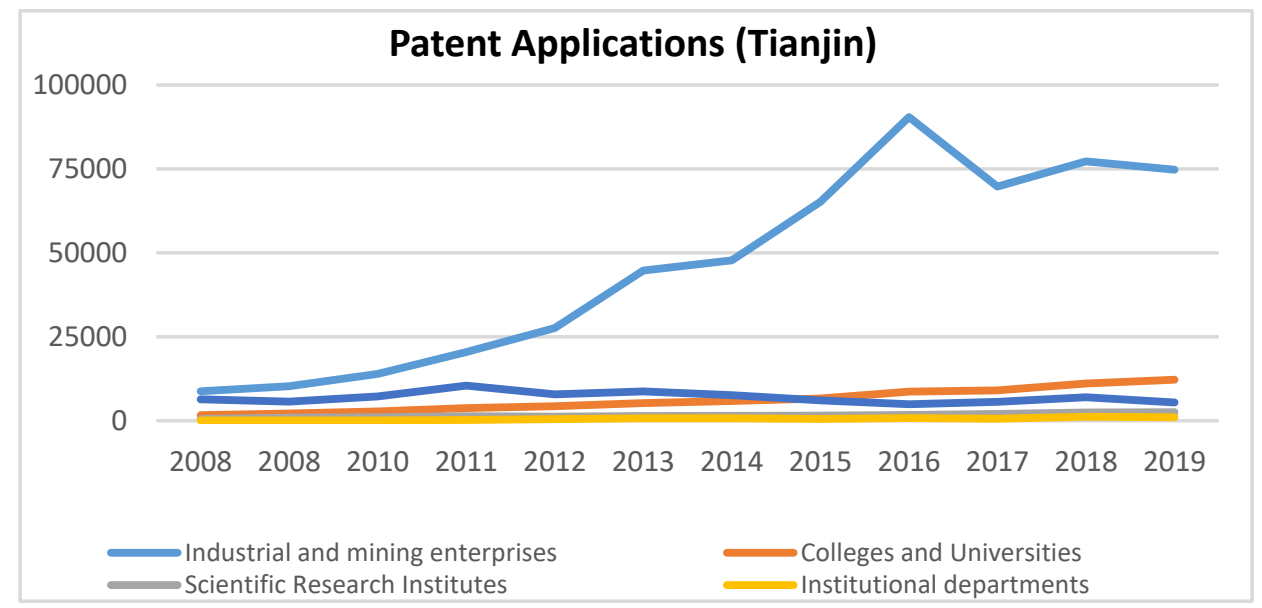

Figure 2. Five Types of Patent Applications in Tianjin

\subsection{Analysis of effective amount of patent application}

Among the 31 provinces and cities in China, the effective number of patents in Tianjin was at a medium level (Table 1). But there was a big gap compared with Beijing, Shanghai, Guangdong and Zhejiang. In 2019, the effective amount of Guangdong Province was more than 11 times that in Tianjin, accounting for $20 \%$ of the national total,
$13 \%$ in Beijing, $12 \%$ in Jiangsu, $7 \%$ in Zhejiang, $6 \%$ in Shanghai and only $2 \%$ in Tianjin, indicating that the effective amount of patent applications in Tianjin needs to be improved.

Table 1. Tianjin Patent Effective Quantity

\begin{tabular}{|l|l|l|l|l|l|l|l|}
\hline 2008 & 13 & 2011 & 15 & 2014 & 13 & 2017 & 12 \\
\hline 2009 & 13 & 2012 & 15 & 2015 & 13 & 2018 & 12 \\
\hline 2010 & 9 & 2013 & 14 & 2016 & 12 & 2019 & 12 \\
\hline
\end{tabular}


In addition to Beijing and Shandong, the provinces with the highest patent effectiveness in China were all southern cities. As a northern city, Tianjin's patent validity is obviously affected by the geographical differences between the north and the south, which was consistent with the characteristics of North-South differences in China's economy, denoting that the level of economic development restricts the maintenance of patent validity in a region.

\section{SHORTCOMINGS IN THE CREATION OF INTELLECTUAL PROPERTY RIGHTS IN TIANJIN}

\subsection{The application level of patent transformation is relatively low in colleges and universities}

The effective patent implementation rate, industrialization rate of colleges and universities were obviously lower than the national average level. At present, the combination of production, education and research is an important mode for the transformation of university achievements. The litigation cases, caused by unclear agreement on intellectual property rights in cooperation, are not uncommon. According to the 2019 Intellectual Property Investigation Report, the biggest obstacle to patent transfer and transformation was the low level of patent technology and the lack of professional teams for patent technology transfer, which exposed the imperfection of patent service system.

\subsection{Lacking a unified intellectual property system between Beijing, Tianjin and Hebei}

The development of intellectual property work cannot be uniformly distributed, and there is no great cooperation within the region. There are great differences in the distribution of various resources, specific administrative regulations, the development of intellectual property work and the construction of law enforcement system in Beijing, Tianjin and Hebei, which leads to the fact that Beijing, Tianjin and Hebei can not become an efficient entirety in the specific work, and there is a certain incompatibility.

\subsection{The cost of infringement of intellectual property rights is low and the cost of safeguarding rights is high.}

Some companies generally have difficulties in obtaining evidence and high costs in obtaining evidence and counterfeiting, making it quite difficult for companies to maintain their rights.[1] As shown in Table 2, since 2018, when major cases of intellectual property rights were investigated and dealt with seriously and quickly, and various activities against infringement continued to be carried out, the number of administrative adjudication cases of patent infringement disputes in Tianjin decreased significantly[2], which decreased to 42 in 2020, but increased to 62 in January 2021, indicating that with the strengthening of law enforcement forces, various false infringements appeared repeatedly, which indirectly reflected the characteristics of low infringement cost.

Table 2. Administrative Adjudication Cases of Patent Infringement Disputes in Tianjin (Unit: Number)

\begin{tabular}{|c|c|c|c|c|c|c|c|}
\hline 2015 & 433 & 2017 & 707 & 2019 & 423 & Jan.2021 & 62 \\
\hline 2016 & 561 & 2018 & 374 & 2020 & 42 & & \\
\hline
\end{tabular}

\section{MEASURES TO PROMOTE THE DEVELOPMENT OF INTELLECTUAL PROPERTY RIGHTS IN TIANJIN}

\subsection{Promote the transformation of innovative achievements from scientific research institutes}

On the one hand, we need to inspire students' innovative thinking through the innovation of teaching mechanisms, improve the ability of university students to combine theory and practice, and strengthen the deep integration of industry[2], academia and research; Encourage the training of IP talents; Introduce education simultaneously, strengthen the construction of full-time team for technology transfer, and build a platform for IP discipline construction; Strengthen the publicity of IP legal knowledge and understanding of the attributes of industry-university-research cooperation transactions, and avoid disputes caused by unclear ownership of IP transactions. On the other hand, the government can give policy and financial support to patent agency service agencies, establish a comprehensive repository of IP professionals[3] so as to increase the evaluation of patent value and the training of IP practitioners, and improve the level of patent services. It is also necessary to improve the IP financial evaluation system to promote the rapid growth of a transparent market.[4]

\subsection{Build a unified intellectual property system}

We can make full use of the advantages of the coordinated development of Beijing, Tianjin and Hebei to integrate and rationally allocate various resources to increase the concentration of production elements, so that we can accelerate the formation of the Bohai Sea economic belt while optimizing and upgrading the industrial structure of Tianjin and fully stimulate the role of Tianjin as the economic centre of the North[5]. This will provide a good economic foundation for scientific and technological innovation and help to enhance the quality of patents so that they can be fully invested in emerging industries such 
as high-end manufacturing, information technology and bio-medicine, thereby nurturing superior enterprises[6].

\subsection{Promote the implementation of intellectual property policies and relevant laws}

We should strengthen the protection of IPR and the implementation of patent supporting policies, safeguard the legitimate rights and interests of patent owners, do not give any counterfeiters who steal patents an opportunity to create a good external environment for patent innovation. We should promote the coordination and linkage between patent systems, provide an efficient and high-quality patent audit system, promote the value transmission of patent achievements to the social field, and look for the contribution of patent policies to social development and cultural prosperity.

\subsection{Establish a perfect legal system and learn from the direct pledge financing mode of "Bank + intellectual property" intellectual property in Beijing}

Intellectual property service institutions, banks and financial institutions will be involved to solve the problems of financing and intellectual property value of small and medium-sized enterprises through a resource sharing platform established by the government. Banks can establish business departments with special IPR, broaden business channels, create financial products linked to IPR and give full play to its value. On the basis of the intellectual property pledge financing model, we also need to use big data and information technology to accurately evaluate intellectual property, promote the transparency of the price of patent achievements, encourage the incubation and construction of a market-oriented and shared evaluation platform, provide guarantee for intellectual property valuation services, and further improve the transaction rate and efficiency of intellectual property transactions. And utilize this to deduce the improvement of technology R\&D and achievement transformation efficiency.

\section{SUMMARY}

Tianjin Intellectual Property Office has strengthened the control of patent "quality" in recent years. The examination and approval of patent applications and the crackdown on patent infringement have been greatly strengthened, which not only purified the patent environment to a certain extent, but also laid a good foundation for the future "high-quality patents". Tianjin intellectual property should build a coordinated and unified intellectual property system in Beijing, Tianjin and Hebei as soon as possible, speed up the construction of intellectual property talents, and complete the efficient transformation of innovative achievements.

\section{ACKNOWLEDGMENT}

The study was supported by "Research on ideas and major measures to improve the creativity of intellectual property in Tianjin in 2035" (Grant No.2021GL008))".

The study was supported by "Research on Tianjin residents' consumption in high quality development stage, China (Grant No. TJYJ20-009)".

The study was supported by "Sense of gain or sense of deprivation: the reference point effect of people's perception of gain and loss, China (Grant No. 71774114)".

\section{REFERENCES}

[1] Li Xiaofeng, Shi Yanhong, Zhao Yini, Ye Runsen. Strategies and countermeasures for Tianjin to improve its intellectual property protection capabilities during the "14th Five-Year Plan" period [J]. Tianjin Economics, 2020(11): 15-19.

[2]2020 Tianjin Intellectual Property White Book.

[3] Tang Jialong, Zhang Lumeng. Analysis of Tianjin Patent Innovation based on the authorization ratio [J]. Anhui Science and Technology, 2020 (04): 27-31.

[4]Li Xiaofeng, Li Jiajun, Cheng Junling. Research on countermeasures for strengthening intellectual property protection and shaping a first-class business environment in Tianjin Binhai New Area[J]. Tianjin Economics, 2021(04): 25-29.

[5] Yuan Zeming, Wang Zijian. Improving intellectual property governance capacity and serving the coordinated development of Beijing, Tianjin and Hebei [J]. Theory and modernization, 2020 (4):56-63.

[6] Ma Yanmin. Analysis of intellectual property financial innovation and risk control. Modern commerce, 2020 (15):118-119. 